\title{
Milled Seed Mix
}

National Cancer Institute

\section{Source}

National Cancer Institute. Milled Seed Mix. NCI Thesaurus. Code C153380.

A nutritional supplement composed of a milled seed mixture, including milled flax, sesame and pumpkin seeds, in sour milk, with potential anti-inflammatory and lipid regulating activities. The seeds contain high levels of fatty acids, including omega-3 and omega- 6 polyunsaturated fatty acids (PUFAs), such as dihomo-gamma-linolenic acid (DGLA), alpha-linolenic acid (ALA), eicosapentaenoic acid (EPA), docosapentaenoic acid (DPA), and docosahexaenoic acid (DHA). Upon administration of the milled seed mix, the intake of PUFAs improves PUFA levels and may favorably change the n-6/n-3 PUFA ratio, decrease triglyceride (TG) levels, and improve body weight. In addition, the active ing redients in the milled seed mix may change the expression of inflammatory markers, such as C-reactive protein (CRP) and pro-inflammatory cytokines, including interleukin-6 (IL-6) and tumor necrosis factor alpha (TNF-a), thereby decreasing inflammation. The milled seed mix may also prevent oxidative stress and affect glycemic control. Tumors cause inflammatory states and unfavorably affect lipid and fatty acid metabolism. 